\title{
Cross Sectional Analysis of Cation Doped Transition Metal Oxide Mesoporous Catalyst Materials
}

Shannon Poges ${ }^{1}$, Biswanath Dutta ${ }^{1}$, Harshul Khanna ${ }^{2}$, Ehsan Moharreri ${ }^{2}$, Mark Aindow ${ }^{2,3}$ and Steven L. Suib $^{1,2,3,4}$

1. Dept. of Chemistry, University of Connecticut, Storrs, CT, USA.

2. Dept. of Materials Science and Engineering, University of Connecticut, Storrs, CT, USA.

3. Institute of Materials Science, University of Connecticut, Storrs, CT, USA.

4. Dept. of Chemical \& Biomolecular Engineering, University of Connecticut, Storrs, CT, USA.

UCT (University of Connecticut) mesoporous transition metal oxide materials are synthesized using an inverse micelle soft template and unique $\mathrm{NO}_{\mathrm{x}}$ chemistry. The resulting aggregated nanoparticles exhibit tunable pore structures, excellent thermal stability, and easily accessible oxidation states which contribute to a wide range of organic catalytic transformations [1]. Among the variety of transition metal oxide systems, mesoporous manganese oxides have proven to be efficient catalysts in the oxidation of alcohols to carboxylic acids. In addition to organic catalysis, there is interest in using the various mesoporous metal oxides in other fields such as energy and advanced separations.

Doping of UCT materials is still in its infancy. Cation promoted mesoporous manganese oxides show increased catalytic activity while maintaining the structural characterization of traditional UCT material. However, one system that has been studied extensively in catalytic reactions involves doping with $\mathrm{Cs}^{+}$ions [2]. The levels of dopants like $\mathrm{Cs}^{+}$can be very small, perhaps 1 in 100 metal ions. In addition there has been some doping with transition metal systems such as iron doped manganese, cobalt doped iron, nickel doped titanium, and similar systems. These materials have interesting magnetic properties. Very few details of the location of these dopant ions are available.

Typically, catalyst morphology is examined using SEM and TEM techniques. A dilute solution drop onto carbon film copper grid sample preparation for TEM is adequate to analyze the electron transparent particles along the edges of the aggregate. FIB has been employed in many cases to analyze cross sections of nanoparticles. This is typically done by first immobilizing the particles in a matrix (epoxy, metal, etc.) or on a substrate in order to obtain an adequate lamella without the loss of material due to structural instability [3-5]. In this work, lamella preparation of a variety of doped UCT materials is performed using an FEI Helios Nanolab 460F1 FIB without the use of bulk matrix immobilization in order to determine if the centers of these aggregates reflect the morphology and pore structure confirmed along the edges. TEM-EDS (FEI Talos S/TEM) of these cross sectioned particles can also be utilized to verify even distribution of detectable dopant amounts in the center of the aggregate [6].

\section{References:}

[1] A. Poyraz et al, Nature Communications 4 (2013) p.2952.

[2] S. Biswas et al, Applied Catalysis B: Environmental 165 (2015) p.731.

[3] C.R. Perrey et al, Journal of Microscopy 214 (2004) p.222.

[4] M.W. Phaneuf in "Introduction to Focused Ion Beams", ed. L.A. Giannuzzi, (Springer, US ) p. 155.

[5] W. Brostow et al., Materials Letters 61 (2007), p. 1333.

[6] This work was supported in part by a research grant from FEI Company under an FEI-UConn 


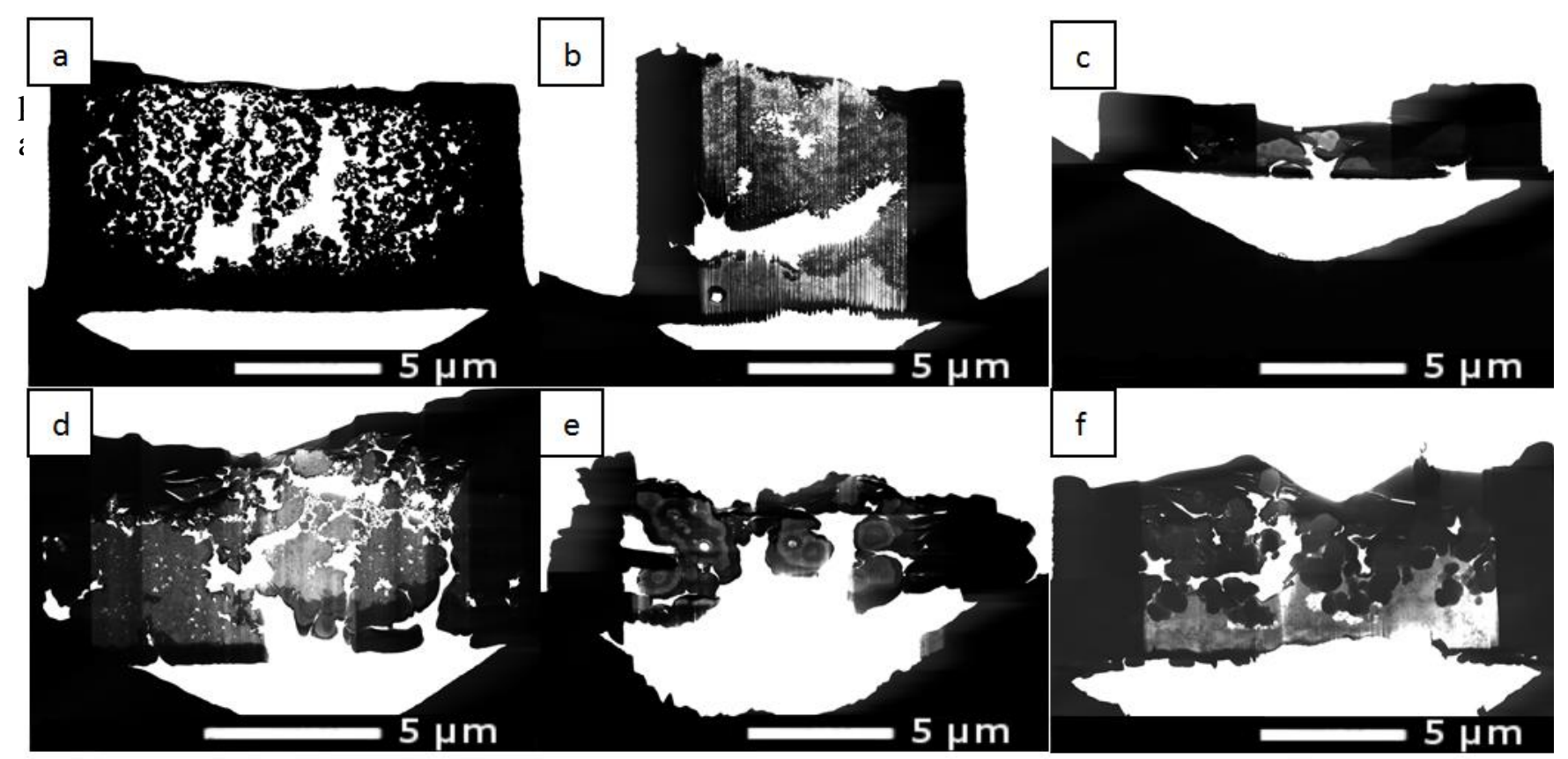

Figure 1. STEM BF images of various doped mesoporous metal oxide lamella; a. Cesium doped manganese oxide, b. Vanadium doped manganese oxide, c. Manganese doped iron oxide, d. Sulfur doped cobalt oxide, e. Cobalt doped manganese oxide, f. Cesium doped manganese oxide.

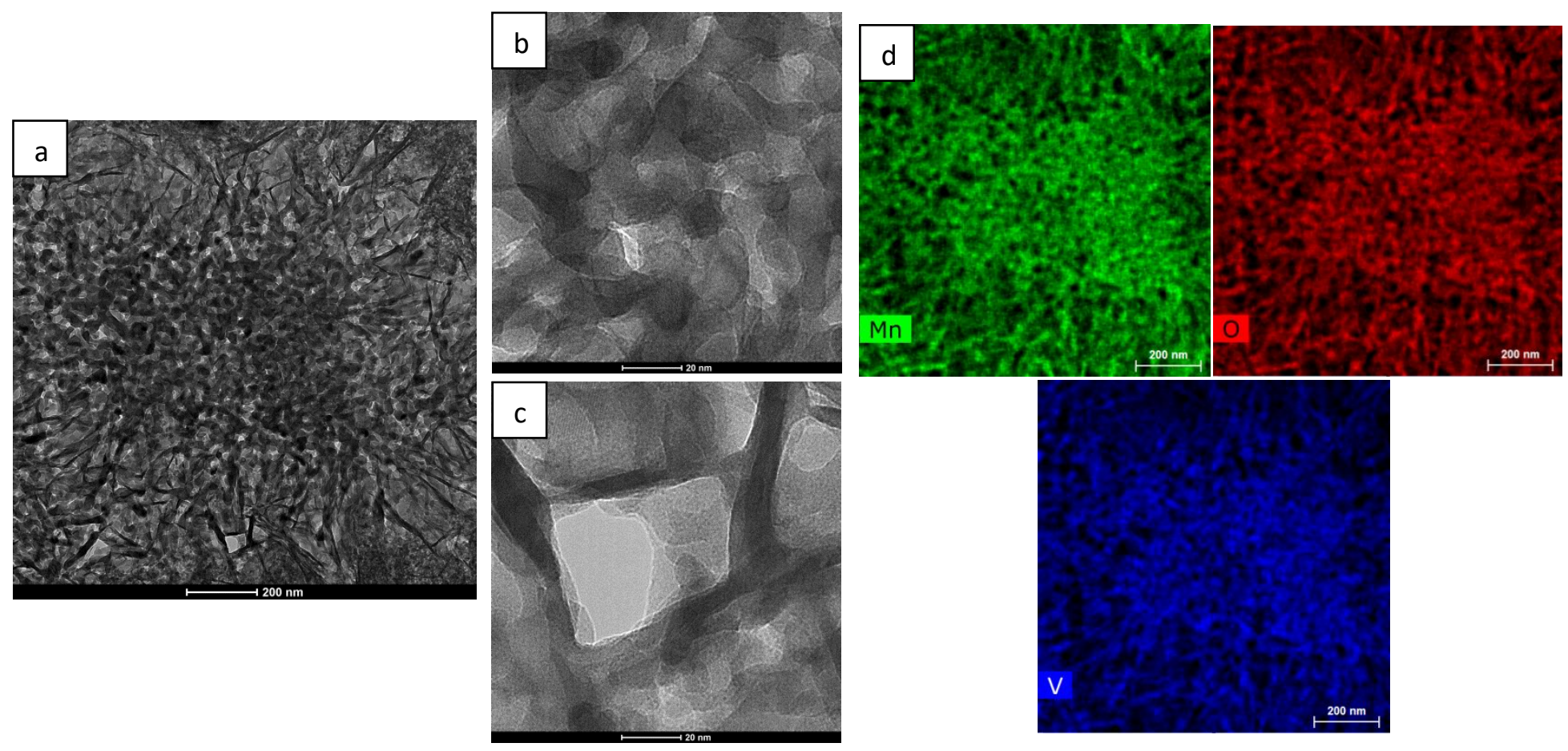

Figure 2. TEM data from FIB prepared vanadium doped mesoporous manganese oxide; a. TEM BF image of one aggregate indicating inconsistency in morphology, b. HRTEM image of the center (mesoporous), c. HRTEM image of the outer sphere (flakey), d. EDS maps indicating even dispersion of vanadium, manganese and oxygen throughout the aggregate. 